\title{
EDITORIAL
}

\section{Transición epidemiológica: la otra cara de la moneda}

\author{
Epidemiological transition. \\ The other side of the coin
}

The model of epidemiological transition proposed by Omram explains the changes in disease patterns in communities. In societies with a high level of development this model has been complemented with the study of the post transition process. In this context, the emergence of allergic diseases, asthma and subsequently, of autoimmune diseases, has reached worrisome proportions in some countries, and no model can explain these changes. The hygiene theory supported by Strachan gives a reasonable explanation to this phenomenon. It postulates that the reduction of early exposure to biological agents, along with an improvement of sanitation conditions, immunizations and medical therapies, causes an asymmetrical immunological response, favoring the expression of Th2 response. The hygiene theory does not fully explain by itself what is happening in developed countries and it is not universally accepted. Chile is experiencing an epidemiological transition from a high burden of infectious diseases to a growing prevalence of non communicable diseases. In a previous similar setting in developed countries, there is some evidence to suspect that asthma, allergic and autoimmune diseases are becoming part of the epidemiological situation of Chile (Rev Méd Chile 2006; 134: 675-8).

(Key words: Autoimmune disease; Chronic disease; Communicable disease; Epidemiology)

$\mathrm{E}^{1}$ modelo explicatorio propuesto por Omram ha sido una buena plataforma de sustentación para comprender los cambios observados en el perfil epidemiológico de enfermedades, en poblaciones con diferente nivel de desarrollo ${ }^{1}$. El carácter universal de tales cambios y su sistematización de acuerdo a diferentes patrones de desarrollo económico, ha permitido también contar con modelos predictivos de amplia aplicación en el mundo subdesarrollado o en proceso de desarrollo.

Correspondencia a: Dr. Gonzalo Valdivia. Departamento de Salud Pública, Escuela de Medicina, Pontificia Universidad Católica de Chile. Marcoleta 434, Casilla 114 D, Santiago Centro, Chile. Teléfono: 354 3038. Fax: 6331840. E mail: valdivia@med.puc.cl.
La teoría de la transición epidemiológica se ha enriquecido a través de la descripción de las características propias del así llamado "período post-transición", caracterizado por avances notables en la contención de las enfermedades crónicas más emblemáticas del período que le precede y la obtención de impensables expectativas de vida de la población en países con elevado nivel de desarrollo ${ }^{2}$. En lo fundamental, los problemas de salud en esta etapa tan avanzada guardan relación con aspectos de calidad de vida, salud mental, conductas y relaciones humanas, malnutrición por exceso, emergencia de nuevas enfermedades transmisibles e influencia del ambiente.

La dinámica de la progresión entre las diversas etapas de la transición epidemiológica es hoy también mejor conocida, observándose diversos 
patrones de instalación de los cambios en cuanto a magnitud y combinación de la carga de enfermedad, conforme las poblaciones modifican sus características de base. Ello depende también de la velocidad con que se producen los cambios demográficos que preceden naturalmente a los de corte epidemiológico.

Este enfoque ha sido de especial utilidad en Chile, donde la información epidemiológica ha explorado estos cambios desde hace al menos 45 años, con estudios regionales en América Latina, encuestas de morbilidad, estudios poblacionales sobre factores de riesgo de enfermedades crónicas y situación nutricional $\mathrm{y}$, en los últimos años, estudios de calidad de vida y la primera encuesta nacional de salud (2003). Paralelamente, en el ámbito de las enfermedades infecciosas se ha contado con información epidemiológica de calidad para una mayor parte de las patologías inmunoprevenibles y de aquellas consideradas como emergentes. Este conocimiento ha permitido ordenar los procesos de planificación sanitaria y reformas sectoriales de país, orientándolos mejor a nuestras necesidades.

Sin embargo, una revisión en los últimos años de lo reportado en los países considerados como de referencia epidemiológica, indica la aparición de problemas de salud que carecen de una base explicatoria tradicional. Ejemplo concreto de esta situación es el reporte de incrementos preocupantes en la prevalencia de enfermedades alérgicas, asma bronquial y fenómenos atópicos, en países en etapas post-transición, llegándose a hablar de verdaderas epidemias de estas enfermedades. Con posterioridad se han reportado cambios en la presentación de enfermedades inflamatorias intestinales crónicas, patologías de corte autoinmune, neuropatías y enfermedades metabólicas como diabetes mellitus tipo 1.

En ausencia de un rebrote de los factores de riesgo clásicos en la fisiopatología de estas enfermedades, la investigación se ha centrado en el estudio de modificaciones de la genética poblacional, en el medio ambiente y en la emergencia de nuevos factores etiológicos que sustituyen a los tradicionales. No sólo la magnitud de estos problemas se modifica. También está cambiando, para algunas enfermedades, su presentación clínica habitual, como ocurre con el asma bronquial.
Cambios radicales, de amplia cobertura y muy dinámicos como los reseñados, estimulan la pesquisa científica de factores comunes de amplia distribución poblacional y la posterior formulación de modelos explicatorios alternativos.

Un modelo atractivo es el derivado de la llamada "Teoría de la Higiene" propuesta por Strachan sobre la base de estudios ecológicos de asociación entre indicadores de carga de exposición a agentes biológicos y diversos modelos de enfermedades crónicas como las ya señaladas ${ }^{3}$. La teoría apoya su plausibilidad planteando que una reducción importante y temprana a la exposición de agentes biológicos vivos de circulación habitual en poblaciones, sumada al adecuado control del macro y microsaneamiento ambiental, impacta en el sistema inmune generando un desbalance en la respuesta inmune de tipo Th1 y Th2, favorable a esta última vía. Esta modificación en el patrón de respuesta inmunológica sería permanente en el tiempo, en la medida que las causas que la explican persistan en el medio ambiente. Aunque no descartable, pero científicamente menos robusto, el componente de genética de poblaciones jugaría un papel marginal en esta teoría. A modo de ejemplo, en países europeos con inmigración relevante desde países del tercer mundo, la incorporación de personas provenientes de ambientes con menor bienestar sanitario y mayores tasas de morbilidad por enfermedades transmisibles, no ha revertido la tendencia al incremento de enfermedades como asma y alergias.

Enfermedades transmisibles como la tuberculosis y el sarampión se asocian inversamente a una mayor prevalencia de asma y enfermedades alérgicas, siendo este efecto más específico para la infección natural respecto de la exposición pasiva vía inmunización programada ${ }^{4,5}$. Otros modelos de exposición antigénica, como ser infecciones virales tempranas en la vida, en especial las de origen respiratorio e intestinal, hepatitis viral, Helicobacter pylori, Toxoplasma gondii, han revelado el mismo patrón de asociación. El tracto digestivo y su aparato linfoide parecen jugar un papel relevante como órgano de transformación de antígenos ambientales y posterior orientación hacia una u otra forma de respuesta inmunológica. Células dendríticas en otros parénquimas 
también están involucradas en esta transformación, como es el caso del aparato respiratorio. La dieta parece jugar un papel importante, considerando el consumo de algunos nutrientes asociados a estilos de vida occidentales ${ }^{6}$.

Estos antecedentes son importantes en nuestro país, que se encuentra en un período de transición epidemiológica avanzada, enfrentando simultáneamente el desafío de la carga de enfermedades crónicas y los rezagos de patologías infecciosas características de etapas pretransicionales ${ }^{7}$.

En este número de la Revista, Luque y cols presentan interesantes antecedentes sobre el cambio en el patrón de enfermedades observado en el periodo post transición epidemiológica en $\mathrm{Chile}^{8}$. Sobre la base de un estudio descriptivo, los autores comparan y analizan la tendencia de enfermedades infecciosas seleccionadas en relación con similar información de algunas enfermedades crónicas, en un período de 53 años. Concluyen señalando una tendencia creciente en la prevalencia de asma bronquial y diabetes mellitus 1, estimando también un efecto similar para la artritis reumatoidea. Se alude en el trabajo a la participación de la teoría de la higiene como modelo explicatorio, al observarse en Chile reducción en la morbilidad de numerosas enfermedades transmisibles, en particular aquellas incluidas en el Programa Ampliado de Inmunizaciones.

\section{REFERENCIAS}

1. OmRam A. The epidemiologic transition: A Theory of the Epidemiology of population change. Milbank Memorial Fund Quarterly 1971; 49: 509-38.

2. Smaшman-Raynor M, Phiшps D. Late stages of epidemiological transition: health status in the developed world. Health \& Place 1999; 5: 209-22.

3. Strachan DP. Hay fever, hygiene, and household size. BMJ 1989; 299: 1259-60.

4. Shaheen S, Barker DJP, Heyes C, Shiell A, Aaby P, HaLl A Et al. Measles and atopy in Guinea-Bissau. Lancet 1996; 347: 1792-6.

5. Matricardi $\mathrm{P}$, Rosmini $\mathrm{F}$, Riondino S, Fortini M, FERRIgno L, Rapicetta $M$ et al. Exposure to
Recientemente, Heaton y cols han comunicado que las vías de respuesta inmunológica Th1/Th2 no son del todo específicas, como se creía en modelos como el asma, documentando la existencia de vías paralelas de activación simultánea en la respuesta Th1/Th2 ${ }^{9}$.

La aceptación de la teoría de la higiene no es universal y aún persisten dudas metodológicas fisiopatológicas, como la recién mencionada, y también de corte epidemiológico ${ }^{9-11}$. La influencia del nivel socioeconómico, de la interacción genética-ambiente, la participación de medidas de salud pública y de los cuidados médicos, son variables complejas de aislar en el análisis de esta teoría.

No obstante, la experiencia reportada desde el mundo desarrollado sobre la irrupción en los perfiles de salud de patologías no tradicionales, asociada a la transición epidemiológica, indica que conviene estudiar anticipadamente la ocurrencia de este fenómeno en nuestro país para disponer así de mejores elementos para enfrentar el advenimiento de este puzzle epidemiológico ${ }^{12}$.

\section{Gonzalo Valdivia C}

Departamento de Salud Pública, Escuela de Medicina, Pontificia Universidad Católica de Chile. Santiago de Chile.

foodborne and orofecal microbes versus airborne viruses in relation to atopy and allergic asthma: epidemiological study. BMJ 2000; 320: 412-7.

6. Weilinand S, von Mutius E, Hüsing A, Asher M, on BEHALF OF THE ISAAC STEERING COMMTTTEE. Intake of trans fatty acids and prevalence of childhood asthma and allergies in Europe. Lancet 1999; 353: 2040-1.

7. Albala C, Vio F. Epidemiological transition in Latin America: the case of Chile. Public Health 1995; 109: 431-42.

8. Luque C, Cisternas F, Araya M. Cambios en el patrón de enfermedad en la postransición epidemiológica en salud en Chile, 1950-2003. Rev Méd Chile 2006; 134: 703-12. 
9. Heaton T, Rowe J, Turner S, Aalberse R, de Kierk N, SURIYAARACHCHI ET AL. An immunoepidemiological approach to asthma: identification of in-vitro $\mathrm{T}$ cell-response patterns associated with different wheezing phenotypes in children. Lancet 2005; 365: 142-9.

10. Brogger J, Eagan T, Eide G, Bakke P, Gulsvik A. Bias in retrospective studies of trends in asthma incidence. Eur Respir J 2004; 23: 281-6.
11. Ambikaipakan S, Lawson J, Rennie D, Dosman J. Stabilization of an increasing Trend in PhysicianDiagnosed Asthma Prevalence in Saskatchewan, 1991 to 1998. Chest 2003; 124: 434-8.

12. VALDivia G. Asma bronquial y enfermedades atópicas como problema emergente de Salud Pública: nuevas hipótesis etiológicas. La experiencia de sociedades desarrolladas. Rev Méd Chile 2000; 128: 339-46. 